\title{
Atypical Imaging Findings of Posterior Reversible Encephalopathy Syndrome in a Child: Case Report and Review of Literature
}

\author{
Guliz Yilmaz $^{\mathrm{a}, \mathrm{c}}$, Taylan Sahin ${ }^{\mathrm{b}}$
}

\begin{abstract}
Posterior reversible encephalopathy syndrome (PRES) is a clinicoradiological entity that is caused by various etiological factors and characterized by headache, confusion, seizure, altered consciousness, visual disturbances and combination of these symptoms. Brain magnetic resonance imaging demonstrates vasogenic edema in the white matter of the parietooccipital lobes typically. Other regions of the brain such as frontal lobe, basal ganglia and deep white matter are affected in patients with atypical PRES. In this report, we present the radiological findings of atypical PRES in a hypertensive child with end stage renal disease and review the current literature related to this situation.
\end{abstract}

Keywords: Posterior reversible encephalopathy syndrome; Hypertension; Magnetic resonance imaging; Computed tomography

\section{Introduction}

Posterior reversible encephalopathy syndrome (PRES) is a clinico-radiological entity characterized by headache, confusion, seizure, altered consciousness, visual disturbances and combination of these symptoms. The diagnosis is made by accompaniment of clinical and radiological findings [1]. The cause of PRES is multifactorial including hypertensive encephalopathy, eclampsia, acute or chronic renal disease, systemic lupus erythematosus and immunosuppressive therapy $[2,3]$. Imaging findings demonstrate vasogenic edema in the

Manuscript accepted for publication March 31, 2014

${ }^{a}$ Radiology, Near East University Hospital, Nicosia, North Cyprus, Turkey

${ }^{\mathrm{b}}$ Anesthesiology and Reanimation, Igdir Public Hospital, Ministry of Health, Igdir, Turkey

${ }^{\mathrm{c}}$ Corresponding author: Guliz Yilmaz, Department of Radiology, Faculty of Medicine, Near East University Hospital, Nicosia, North Cyprus,

Turkey. Email: glz.yilmaz@hotmail.com

doi: http://dx.doi.org/10.14740/ijcp142w white matter of the parietooccipital lobes on brain magnetic resonance imaging (MRI). Other regions of the brain such as frontal lobe, basal ganglia and deep white matter are affected in patients with atypical PRES [4-6]. We present the radiological findings of atypical PRES case and the current literature related to this object.

\section{Case Report}

A 16-year-old girl who was diagnosed with end stage renal disease (ESRD) was brought to the emergency department with severe headache, altered consciousness and vomiting. She was initially diagnosed as membranoproliferative glomerulonephritis 1 year ago.

Her blood pressure was $180 / 120 \mathrm{mmHg}$ at the emergency department and physical examination was normal except for generalized edema. The white blood cell count was 19.8 $\times 10^{3} / \mu \mathrm{L}$, protein: $3.9 \mathrm{~g} / \mathrm{dL}$ and albumin: $1.7 \mathrm{~g} / \mathrm{dL}$ as abnormal results in her laboratory findings. In her urine analysis, density was measured $1,015 \mathrm{pH}$ and proteinuria and hematuria were present.

She had generalized tonic clonic seizures lasting in 5 $\min$. The seizures were stopped spontaneously in the following days.

Brain computed tomography (CT) showed effacement of sulci in the left cerebral hemisphere and hypodense region in the subcortical and deep white matter of the occipitoparietal lobe on the day of the initial seizure (Fig. 1A, B). Brain MRI demonstrated asymmetrical vasogenic edema as hyperintense signal on T2 weighted and fluid attenuated inversion recovery (FLAIR) sequences in the subcortical region of the left occipitoparietal and temporal lobe, right posterior frontal lobe and a focal milimetric area of the left posterior frontal lobe (Fig. 2A-D) No abnormality was seen in the posterior fossa or the basal ganglia. Diffusion weighted imaging (DWI) and apparent diffusion coefficients (ADC) maps showed no restricted diffusion but T2 shine through effect (Fig. 2E, F). The diagnosis was thought to be atypic PRES in the patient who was under immunosuppressive therapy with clinical and radiological findings.

The patient was referred to the pediatric nephrology 


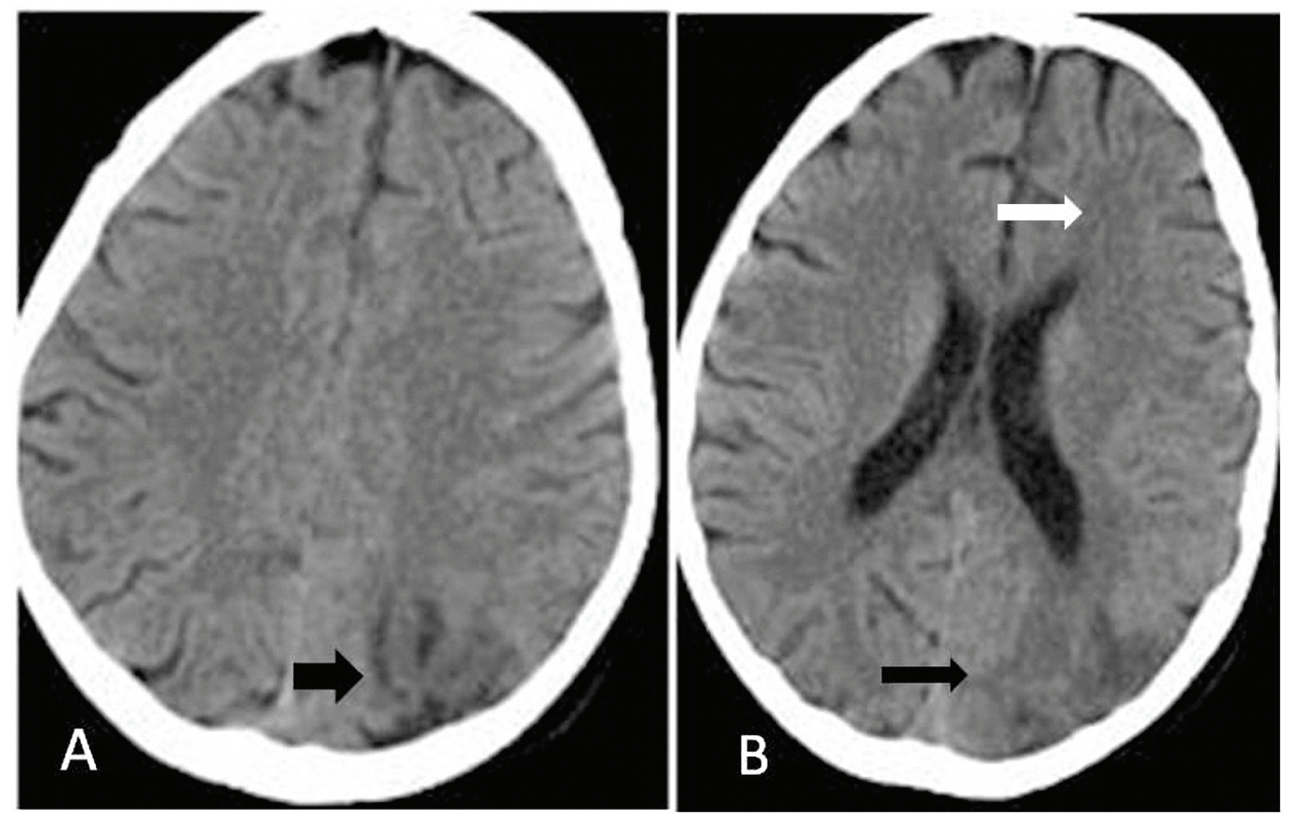

Figure 1. (A, B) Axial CT scan of brain showed effacement of sulci in the left cerebral hemisphere (white arrow) and hypodense region in the subcortical and deep white matter of the occipitoparietal lobe (black arrow).

clinic due to impaired general condition although the blood pressure was stable in normal limits with anti-edema, anticonvulsant and renal support treatment in the intensive care unit.

\section{Discussion}

PRES is a clinico-radiological entity characterized by severe headaches, confusion, visual disturbances, seizures, altered consciousness and combination of these symptoms with imaging findings of vasogenic edema in a posterior circulation distribution $[1,7]$. PRES, known as posterior reversible encephalopathy or reversible posterior leukoencephalopathy was first described in 1996 by Hinchey et al [1]. However, it can be irreversible and is often not confined to either the white matter or the posterior regions of the brain; alternative terms such as focal acute cerebral edema or potential reversible encephalopathy have been suggested $[6,8]$.

The cause of PRES is controversial and multifactorial including hypertensive encephalopathy, eclampsia, acute or chronic renal disease, systemic lupus erythematosus, thrombotic thrombocytopenic purpura, collagen vascular disease and immunosuppressive therapy $[2,3]$.

The pathophysiology of PRES is unclear. Two theories have been suggested. In the first hypothesis, it has been suggested that cerebral vasospasm contributes to ischemia and cytotoxic edema in regions of the arterial border zone due to acutely increased blood pressure. The second more recent hypothesis depends on loss of autoregulation. Autoregula- tion maintains constant cerebral blood flow by the sympathetic system. As the elevation of the blood pressure, arteriolar vasoconstriction obstructs cerebral hyperperfusion by the sympathetic system. This autoregulation does not work over the threshold values of the blood pressure. Eventually vasodilation occurs in hypertension resulting brain hyperperfusion. This leads to breakdown of the blood-brain barrier allowing extravasation of fluid and blood products into the interstitium cause vasogenic edema. The posterior regions of the brain are more affected due to relatively poor sympathetic system in the posterior circulation $[9,10]$.

It is thought that the sudden increases in blood pressure which is the most frequently encountered etiologies in the literature leads to disruption of the autoregulatory mechanisms resulting in a breakdown of the blood-brain barrier [1, 11]. ESRD and hypertension might have caused PRES in our patient's attack.

The most common abnormal imaging finding is focal regions of symmetric hemispheric vasogenic edema involving the white matter in the bilateral parietooccipital lobes of the cerebral hemispheres [1]. Although PRES typically affects the parietal and occipital lobes, other regions of the brain such as frontal lobe, basal ganglia, cortex and deep white matter are affected in atypical PRES [4-6, 12]. The lesions of PRES are best visualized with MRI, which is able to show even small lesions. MRI lesions are hyperintense on T2W and FLAIR sequences and hypointense on T1W sequences. DWI and ADC demonstrate the vasogenic edema certainly. The edema usually completely reverses $[3,11,13]$. The frontal lobe involvement was the atypical lesion found in our 

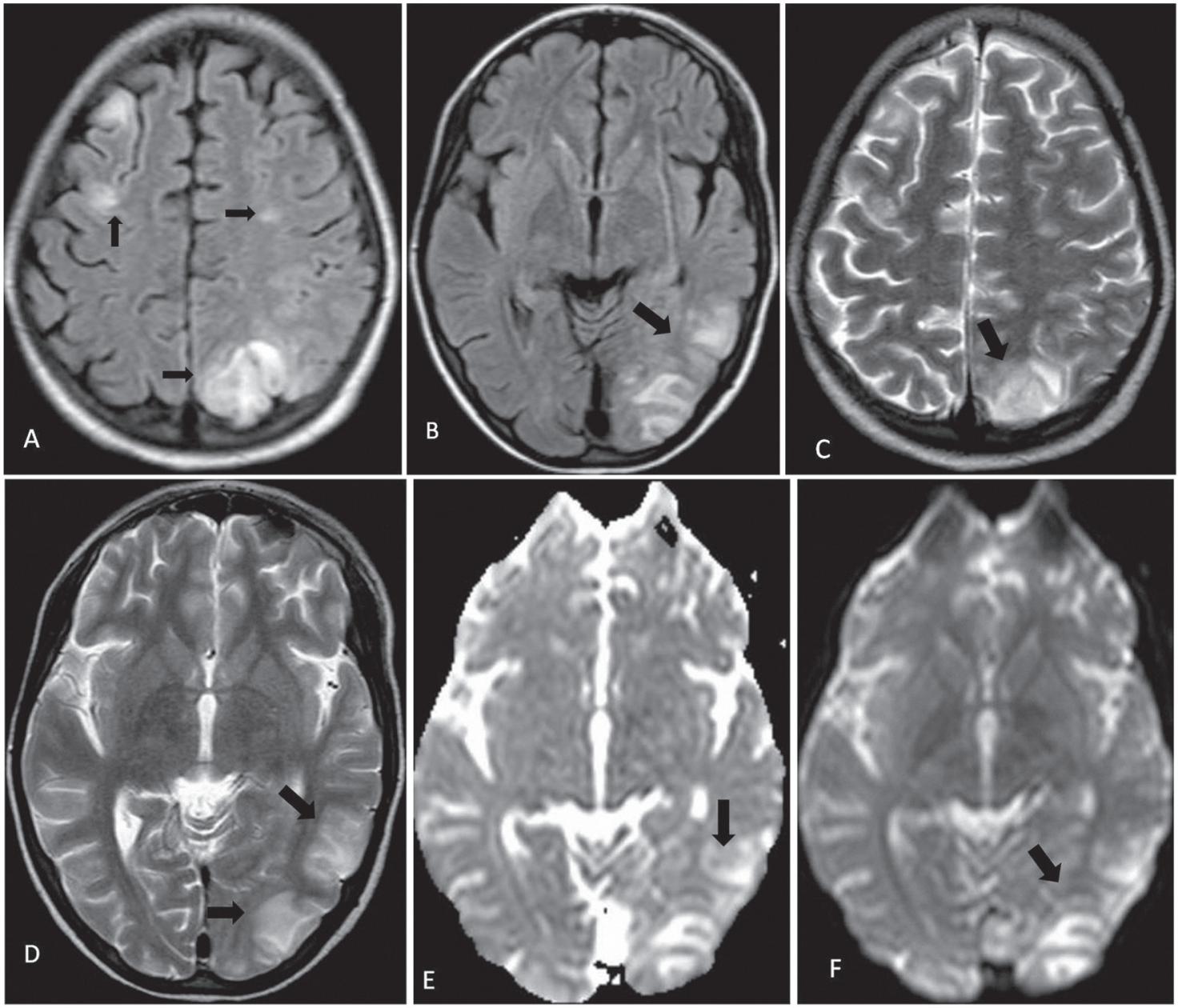

Figure 2. (A-D) Brain MRI showed increased T2 signal in the subcortical region of the left occipitoparietal lobe, right posterior frontal lobe and small area of the left posterior frontal lobe on T2 weighted and FLAIR sequences (black arrow). (E, F) DWI and ADC maps showed no restricted diffusion but T2 shine through effect (black arrow).

patient.

The differential diagnoses of PRES are posterior lobes infarcts, cerebral venous thrombosis, encephalitis, cerebral involvement of vasculitis and encephalopathy [14]. In our case, the venous or ischemic infarcts were excluded by MRI. The other situations were excluded by the clinical follow-up studies.

This syndrome should be recognized immediately in order to prevent long-term neurological sequelae. Treatment includes antihypertensive and antiepileptic therapy and stopping the etiologic agent in the case of drug reaction. Radiological and clinical abnormalities can completely resolve by obtaining autoregulation again [9].

\section{Conclusion}

In conclusion, PRES is a rare complication of different diseases in childhood. Recognition of PRES and imaging by MRI can be helpful to prevent the irreversible brain compli- cations in patients with ESRD who have neurologic findings.

\section{Disclosure}

The authors declare no conflict of interest.

\section{References}

1. Hinchey J, Chaves C, Appignani B, Breen J, Pao L, Wang A, Pessin MS, et al. A reversible posterior leukoencephalopathy syndrome. N Engl J Med. 1996;334(8):494500 .

2. Brubaker LM, Smith JK, Lee YZ, Lin W, Castillo M. Hemodynamic and permeability changes in posterior reversible encephalopathy syndrome measured by dynamic susceptibility perfusion-weighted MR imaging. AJNR Am J Neuroradiol. 2005;26(4):825-830. 
3. Mukherjee P, McKinstry RC. Reversible posterior leukoencephalopathy syndrome: evaluation with diffusiontensor MR imaging. Radiology. 2001;219(3):756-765.

4. Ahn KJ, You WJ, Jeong SL, Lee JW, Kim BS, Lee JH, Yang DW, et al. Atypical manifestations of reversible posterior leukoencephalopathy syndrome: findings on diffusion imaging and ADC mapping. Neuroradiology. 2004;46(12):978-983.

5. Kitaguchi H, Tomimoto H, Miki Y, Yamamoto A, Terada K, Satoi H, Kanda M, et al. A brainstem variant of reversible posterior leukoencephalopathy syndrome. Neuroradiology. 2005;47(9):652-656.

6. Bianco F. Reversible posterior leukoencephalopathy syndrome: a changing concept. Neuroradiology. 2005;47(9):703-704; author reply 705.

7. Lamy C, Oppenheim C, Meder JF, Mas JL. Neuroimaging in posterior reversible encephalopathy syndrome. $\mathrm{J}$ Neuroimaging. 2004;14(2):89-96.

8. Narbone MC, Musolino R, Granata F, Mazzu I, Abbate M, Ferlazzo E. PRES: posterior or potentially reversible encephalopathy syndrome? Neurol Sci. 2006;27(3):187189.

9. Pande AR, Ando K, Ishikura R, Nagami Y, Takada Y,
Wada A, Watanabe Y, et al. Clinicoradiological factors influencing the reversibility of posterior reversible encephalopathy syndrome: a multicenter study. Radiat Med. 2006;24(10):659-668.

10. Schwartz RB, Mulkern RV, Gudbjartsson H, Jolesz F. Diffusion-weighted MR imaging in hypertensive encephalopathy: clues to pathogenesis. AJNR Am J Neuroradiol. 1998;19(5):859-862.

11. Onder AM, Lopez R, Teomete U, Francoeur D, Bhatia R, Knowbi O, Hizaji R, et al. Posterior reversible encephalopathy syndrome in the pediatric renal population. Pediatr Nephrol. 2007;22(11):1921-1929.

12. Covarrubias DJ, Luetmer PH, Campeau NG. Posterior reversible encephalopathy syndrome: prognostic utility of quantitative diffusion-weighted MR images. AJNR Am J Neuroradiol. 2002;23(6):1038-1048.

13. Ay H, Buonanno FS, Schaefer PW, Le DA, Wang B, Gonzalez RG, Koroshetz WJ. Posterior leukoencephalopathy without severe hypertension: utility of diffusion-weighted MRI. Neurology. 1998;51(5):1369-1376.

14. Ringelstein EB, Knecht S. Cerebral small vessel diseases: manifestations in young women. Curr Opin Neurol. 2006;19(1):55-62. 